\title{
The effect of motility and cell-surface polymers on bacterial attachment
}

\author{
Hisao Morisaki, ${ }^{1}$ Shinichi Nagai, ${ }^{1}$ Hiroyuki Ohshima, ${ }^{2}$ Eiko Ikemoto ${ }^{3}$ \\ and Kazuhiro Kogure ${ }^{3}$
}

Author for correspondence: H. Morisaki. Tel: +8177 561 2767. Fax: +81 775612659.

e-mail:morisaki@se.ritsumei.ac.jp

\footnotetext{
1 Faculty of Science and Engineering, Ritsumeikan University, 1-1-1 Nojihigashi, Kusatsu 525-8577, Japan

2 Faculty of Pharmaceutical Sciences, Science University of Tokyo, Shinjuku-ku, Tokyo 162-0826, Japan

3 Ocean Research Institute, University of Tokyo, Minamidai, Nakano, Tokyo 164-8639, Japan
}

\begin{abstract}
Recently it was shown that motility of Vibrio alginolyticus facilitated cell attachment to glass surfaces. In the present study the same relationship between motility and cell attachment was confirmed for Alcaligenes and Alteromonas spp. These findings clearly answer a long-standing question: does motility facilitate attachment? However, they are contradictory to a general view on cell attachment that the energy barrier due to electrostatic repulsion between negatively charged bacterial cells and a glass surface is much greater than both the thermal kinetic energy of the bacterial cell and the bacterial swimming energy. It is shown that the energy barrier becomes far less than that usually estimated when bacterial cells are rich in polymers at their surfaces. This finding reasonably explains the dependence of bacterial attachment rate on cell motility and demands reconsideration of the mechanism of bacterial attachment.
\end{abstract}

Keywords: cell attachment, motility, surface polymers, electrostatic repulsion, soft
particle
Keywords: cell attachment, motility, surface polymers, electrostatic repulsion, soft
particle

\section{INTRODUCTION}

The marine bacterium Vibrio alginolyticus can swim in water by rotating a polar flagellum driven by $\mathrm{Na}^{+}$ motive force. Thus, the cell swimming speed is dependent on $\mathrm{Na}^{+}$concentration. Recently, Kogure et al. (1998) reported that the attachment rate of V. alginolyticus cells to a glass surface is dependent on $\mathrm{Na}^{+}$ concentration, i.e. the swimming speed of the cells $\left(\mathrm{K}^{+}\right.$ was added to keep the ionic strength constant). This is also the case for other bacterial strains, as revealed in the present study.

These findings clearly answer a long-standing question: does motility facilitate attachment? However, another unsolved problem remains: how the bacteria can overcome the energy barrier, estimated to be about $10^{2}$ times greater than the thermal kinetic energy of the bacterial cell or about $10^{6}$ times greater than the cell swimming energy according to the DLVO theory (Marshall et al., 1971), due to electrostatic repulsion between the cells and glass (commonly both of them are negatively charged).

Abbreviations: EPM, electrophoretic mobility; SSW, synthetic seawater.
Ohshima and co-workers (Oshima et al., 1993; Oshima, 1994, 1995) pointed out that the electrokinetic behaviour of soft, polyelectrolyte-covered colloidal particles is different from that of 'rigid' particles having no polymer, and proposed a new theory which can analyse the surface polymers on soft particles. This theory was applied to bacterial cells (typical examples of soft particles) and the polymer layers on bacterial cells were characterized (Takashima \& Morisaki, 1997; Bos et al., 1998).

The aim of this study was to analyse the surface polymers on the cells of $V$. alginolyticus by the 'softparticle' theory and to clarify the effect of polymers on the interaction between the cells and glass surfaces. We report here that the energy barrier due to the electrostatic repulsion between bacterial cells and a glass surface becomes much smaller than that usually expected, even less than the thermal kinetic energy, when the bacteria are rich in polymers at the cell surfaces. This finding reasonably explains the dependence of the cell attachment rate on the swimming speed. Moreover, it provides us with a new way to understand the interaction between surfaces when either or both of them have a polymer layer. Our findings demand reconsideration of the mechanism of bacterial attachment and subsequent biofilm formation causing various problems such as 
food contamination, ship biofouling and pipeline corrosion.

\section{METHODS}

Organisms and culture conditions. Three strains of Vibrio alginolyticus, YM4 ( $\left.\operatorname{Pof}^{+} \mathrm{Laf}^{-}\right)$, YM18 ( $\left.\mathrm{Pof}^{-} \mathrm{Laf}^{-}\right)$and NMB94 $\left(\right.$ Pof $^{+}$Laf $^{-}$Mot $^{-}$, were kindly supplied by Dr. H. Homma, Nagoya University, Japan. The cells of these strains were cultured in quarter-strength ZoBell 2216E medium prepared with synthetic seawater (SSW) containing $\mathrm{NaCl} 23.4 \mathrm{~g}, \mathrm{KCl}$ $0.8 \mathrm{~g}, \mathrm{MgSO}_{4} .7 \mathrm{H}_{2} \mathrm{O} 4 \mathrm{~g}, \mathrm{CaCl}_{2} 0.2 \mathrm{~g}, \mathrm{KBr} 100 \mathrm{mg}$, $\mathrm{SrCl}_{2} \cdot 6 \mathrm{H}_{2} \mathrm{O} 26 \mathrm{mg}, \mathrm{H}_{3} \mathrm{BO}_{3} 20 \mathrm{mg}$ and HEPES $4.76 \mathrm{~g}$ per litre of distilled water ( $\mathrm{pH} 7 \cdot 5)$. Full-strength ZoBell 2216E medium contains Polypepton (5 $\mathrm{g} \mathrm{l}^{-1}$, Japan Pharmaceuticals) and yeast extract ( $1 \mathrm{~g} \mathrm{l}^{-1}$, Difco) as organic substrates. Alcaligenes sp. 172 and 201 and Alteromonas sp. WB30 were cultured in quarter-strength ZoBell 2216E medium prepared with a mixture of $80 \%$ SSW and $20 \%$ distilled water. Pseudomonas aeruginosa was cultured in quarter-strength ZoBell 2216E medium prepared with distilled water. Cells of overnight cultures at $25^{\circ} \mathrm{C}$ were centrifuged at $5000 \mathrm{~g}$ for $5 \mathrm{~min}$ at $10^{\circ} \mathrm{C}$. After washing twice with SSW or $400 \mathrm{mM} \mathrm{NaCl}$ aqueous solution (in the case of electrophoretic mobility measurement; see below), the cells were suspended in the solution.

Electrophoretic mobility (EPM) measurement. The EPM of the cells was measured at $25^{\circ} \mathrm{C}$ with system 3000 (Pen Kem). The cell suspension in $400 \mathrm{mM} \mathrm{NaCl}$ solution was mixed $(1: 39, \mathrm{v} / \mathrm{v})$ with a phosphate buffer solution $(\mathrm{pH} 7 \cdot 4)$. The ionic strength of the buffer was varied, while its osmotic pressure was maintained constant, by changing the ratio of $0 \cdot 154 \mathrm{M}$ phosphate buffer, a sucrose solution $(0.5 \mathrm{M})$ and distilled water.

Attachment measurement. Precleaned cover glasses $(24 \times 32 \mathrm{~mm}$, NEO no. 1; Matsunami, Osaka, Japan) were used as the attachment substratum. They were washed by ultrasonication and dried before being packed and shipped by the manufacturer. We compared several methods of precleaning (two kinds of detergent and $\mathrm{HCl} /$ ethanol treatment), but there was virtually no difference in the degree of attachment. Therefore we used the cover glasses without any pretreatment. One piece of cover glass was placed in a cell suspension $(10 \mathrm{ml})$ in a test tube $(30 \times 60 \mathrm{~mm})$ with shaking at $100 \mathrm{r} . \mathrm{p} . \mathrm{m}$. on a rotary shaker at $25^{\circ} \mathrm{C}$. After $1 \mathrm{~h}$, the glass was taken out and washed twice by dipping and shaking it gently in SSW ; only the cells whose attachment energy is greater than the energy of the applied shear force during the washing procedure would remain. One drop of DAPI (4',6-diamidino2-phenylindole) solution (approx. $1 \mu \mathrm{g} \mathrm{ml}^{-1}$ ) was placed on the glass surface, which was observed with a fluorescence microscope (Olympus BH-2) within $30 \mathrm{~min}$. Cells in at least 10 randomly chosen fields were counted at a magnification of $\times 1000$ and the counts averaged. Assays for each experimental condition were repeated twice to confirm the reproducibility of the results.

Swimming speed measurement. Twenty microlitres of cell suspension (preincubated for $30 \mathrm{~min}$ in SSW of the particular composition to be used) was placed onto a glass slide, and the swimming speed of planktonic cells was measured as follows. The movements of planktonic cells were immediately recorded with an inverted microscope (Diaphot 300; Nikon) at a magnification of $\times 400$ and a high-speed video camera (HSV500 HR; Nac) for $2 \mathrm{~min}$. The focus was set at the top of the surface layer. The images obtained were analysed by using Movias 2D software (Nac). From one frame, 20 cells were randomly chosen, regardless of their motility. The distance travelled in $40 \mathrm{~ms}$ was consecutively measured for up to $1 \mathrm{~s}$. When a cell moved out of the layer being focused on, measurement was stopped and the data that had been collected until this time point were used. Assays were repeated twice.

\section{RESULTS AND DISCUSSION}

\section{Effect of polymers on the electrokinetic behaviour of bacterial cells}

Polymers at the surface of colloidal particles greatly affect the electrokinetic behaviour of the particles whether the polymers are electrically neutral (Hjerten, 1967) or charged (Ohshima et al., 1993; Makino et al., 1994). Bacterial cells usually have various charged polymers at their surfaces. Thus, we must take into account the presence of polymers having a non-zero charge density to evaluate the surface characteristics of bacterial cells, e.g. electric surface potential, from the electrokinetic behaviour of the cells. The effect of polymers can be estimated from the dependence of the EPM of colloidal particles on the ionic strength of the medium.

We measured the EPM of $V$. alginolyticus cells in medium at different ionic strengths. The EPM of the three strains of $V$. alginolyticus changed along with the ionic strength of the medium as shown in Fig. 1. At a low ionic strength the EPM became slightly less negative with the increment in the ionic strength, but the EPM levelled off at a high ionic strength. This pattern of EPM change is totally different from that usually expected, as follows. For colloidal particles, including bacterial cells,

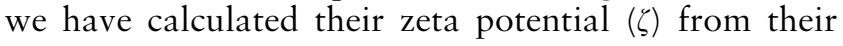

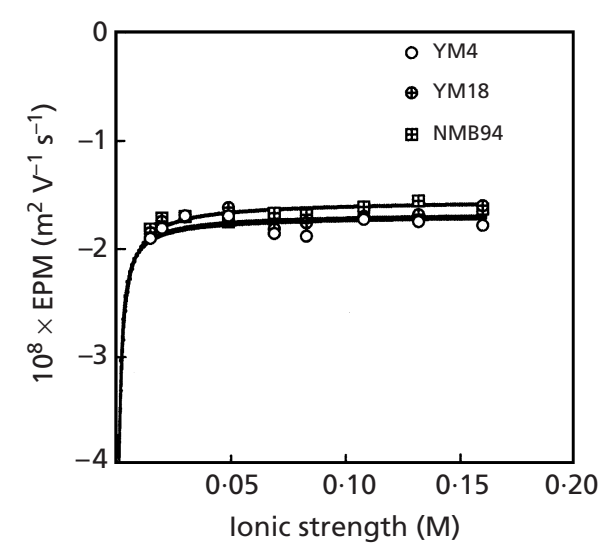

Fig. 1. Changes in the EPM of the three $V$. alginolyticus strains as a function of ionic strength. The ratio of $0.154 \mathrm{M}$ phosphate buffer $(\mathrm{pH} 7.4)$, a sucrose solution $(0.5 \mathrm{M})$ and distilled water was changed to vary the ionic strength. The ionic strengths used for the EPM measurements were substantially smaller than those of SSW (ionic strength approx. 0.5 M). The measurements were repeated twice independently for each strain and the mean values were plotted; the mean of the standard error was $0.026 \times 10^{-8} \mathrm{~m}^{2} \mathrm{~V}^{-1} \mathrm{~s}^{-1}$. Solid lines show the theoretical curves calculated from equation (2) by using the following parameters; $N=-0.0038(\mathrm{M}), 1 / \lambda=6.44(\mathrm{~nm})$ for strain YM4 (Pof $^{+}$Laf $\left.^{-}\right) ; N=-0.0050(\mathrm{M}), 1 / \lambda=5.43(\mathrm{~nm})$ for strain YM18 $\left(\right.$ Pof $^{-}$Laf $\left.^{-}\right) ; N=-0.0055(\mathrm{M}), 1 / \lambda=5.13(\mathrm{~nm})$ for strain NMB94 $\left(\right.$ Pof $^{+}$Laf $^{-}$Mot $\left.^{-}\right)$. 
electrophoretic mobility $(\mu)$ by the Smoluchowski equation: $\mu=\varepsilon_{\mathrm{r}} \varepsilon_{0} \zeta / \eta$, where $\varepsilon_{\mathrm{r}}$ is the relative permittivity of the medium in which the cells suspended, $\varepsilon_{0}$ is the permittivity of a vacuum and $\eta$ is the viscosity of the medium. We have also used the equation $\Psi_{0}=\sigma / \varepsilon_{\mathrm{r}} \varepsilon_{0} \kappa$ to relate the surface potential $\left(\Psi_{0}\right)$ of the particles to the surface charge density $(\sigma)$, where $\kappa$ is the Debye-Hückel parameter, depending mainly on the square root of electrolyte concentration. Combination of these equations by approximating the zeta potential with the surface potential gives:

$\mu=\sigma / \eta \kappa$

Equation (1) predicts that the EPM will decrease proportionally to the square root of the increasing ionic strength for particles like bacteria whose surface charge is due to ionization of functional groups. However, the observations for various kinds of biological cells (van Loosdrecht et al., 1987; Kawahata et al., 1990; Rijnaarts et al., 1995; Sonohara et al., 1995; Takashima \& Morisaki, 1997) hardly obey this prediction. Although it has been pointed out that surface conductance on bacterial cells may have a considerable effect on the electrophoretic mobility of the cells at a low ionic strength (van der Wal et al., 1997), the changing pattern of EPM as shown in Fig. 1, i.e. gradually approaching to a non-zero value with increasing ionic strength, cannot be explained by the conductance effect.

Ohshima $(1994,1995)$ has pointed out that the Smoluchowski equation cannot be applied to 'soft' particles having polymers at their surfaces: the equation seems to be limited to 'rigid' particles having no polymer. He solved the problem concerning 'soft' particles whose EPM approaches a non-zero value with increasing ionic strength by presenting a theory formulated as follows.

$\mu=\left(\frac{\varepsilon_{\mathrm{r}} \varepsilon_{0}}{\eta}\right)\left(\frac{\Psi_{0} / \kappa_{\mathrm{m}}+\Psi_{\mathrm{DON}} \lambda}{\left(1 / \kappa_{\mathrm{m}}+1 / \lambda\right.}\right)+\frac{z e N}{\eta \lambda^{2}}$

where $\Psi_{\text {DON }}$ is the Donnan potential of the polymer layer, $\kappa_{\mathrm{m}}$ can be interpreted as the Debye-Hückel parameter of the polymer layer, $\lambda$ is a parameter whose reciprocal has the dimension of length corresponding to a softness of the polymer layer, $z$ is the valence of charged group in the polymers, $e$ is the electric unit charge and $N$ is the density of the charged groups in the polymer layer.

Ohshima's theory can explain the EPM change along with the ionic strength by considering the presence of charged polymer layer at the cell surface. We can characterize the polymer layer by determining its charge density and softness to fit the theoretical curve from equation (2) to the actual EPM change as shown in Fig. 1. In equation (2), $\Psi_{0}, \Psi_{\text {DON }}$ and $\kappa_{\mathrm{m}}$ are functions of ionic strength, and the value defined by the first term in this equation decreases along with the increment in the ionic strength. However, the second term, $z e N / \eta \lambda^{2}$, gives a constant value regardless of the change in ionic strength. Hence, the EPM levels off to a non-zero value defined by the second term at a high ionic strength (Ohshima, 1994, 1995). The theory also gives us the

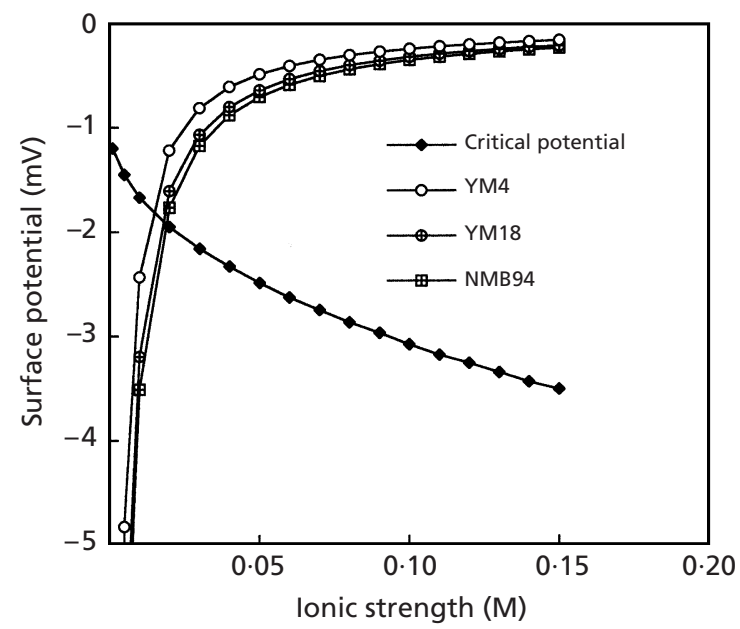

Fig. 2. Surface potential of three $V$. alginolyticus strains calculated from Ohshima's theory as a function of ionic strength. The cell-surface potential where the energy barrier between the cells and glass was equal to the cell thermal kinetic energy at $25^{\circ} \mathrm{C}$ was plotted as the critical potential. The same parameters $(-15 \mathrm{mV}$ as the surface potential of glass; $5 \times 10^{-22} \mathrm{~J}$ as Hamaker's constant) and equation as used by Marshall et al. (1971), except the radius of cells, were used for the calculation of the barrier by the DLVO theory. The equation was as follows. Total interaction energy $=$ repulsion energy + attraction energy $=\pi \varepsilon_{\mathrm{r}} \varepsilon_{0} \mathrm{a}\left[\left(\Psi_{1}+\Psi_{2}\right)^{2}\right.$ $\left.\ln \left(1+\mathrm{e}^{-\kappa h}\right)+\left(\Psi_{1}-\Psi_{2}\right)^{2} \ln \left(1-\mathrm{e}^{-\kappa h}\right)\right]-A a / 6 h$, where $\varepsilon_{\mathrm{r}}$ is the relative permittivity of the medium in which the cells suspended, $\varepsilon_{0}$ is the permittivity of a vacuum, $a$ is the radius of the bacterial cell, $\Psi_{1}$ is the surface potential of glass, $\Psi_{2}$ is the surface potential of the bacterial cell, $\kappa$ is the inverse Debye-Hückel length, $A$ is Hamaker's constant and $h$ is the distance of closest approach between glass and bacterial cell. The cells of the three strains of $V$. alginolyticus were $0.74 \times 2.38$, $0.77 \times 2.68$ and $0.81 \times 2.54 \mu \mathrm{m}$ (mean of two independent experiments for 10 cells; standard error was less than $0.17 \mu \mathrm{m}$ for the minor axis and $0.33 \mu \mathrm{m}$ for the major axis) for strains YM4, YM18 and NMB94, respectively. By taking the mean values and the standard errors of the minor axis into account (the barrier is lower for the cells directing a side of smaller radius toward the glass surface), the value of $0.5 \mu \mathrm{m}$ as the cell radius was used for the barrier calculation.

electric potential at the outermost fringe of the polymer layer, i.e. the surface potential. The surface potential of $V$. alginolyticus obtained from this theory (Fig. 2) was surprisingly small compared to that calculated by putting the EPM value of the cells shown in Fig. 1 into the Smoluchowski equation: the potential from the Smoluchowski equation ranged from $-20 \cdot 2$ to $-24.4 \mathrm{mV}$ in the ionic strength range 0.01 to $0 \cdot 15 \mathrm{M}$. Fig. 2 also shows that, above a critical ionic strength, the bacterial cells can surmount the energy barrier between the cells and glass, because the cell-surface potential is so small as to make the energy barrier less than the cell thermal kinetic energy.

\section{Relationship between cell motility and attachment rate}

The above consideration leads to the conclusion that we have overestimated the height of the energy barrier 


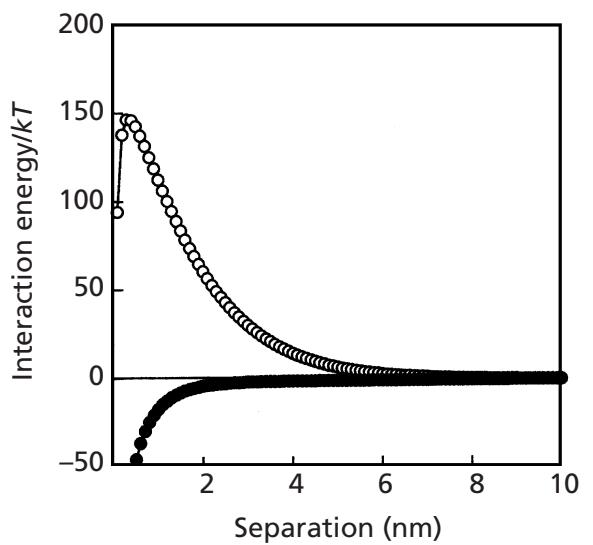

Fig. 3. Interaction energy between microbial cells and a glass surface as a function of separation between them at the ionic strength $0.05 \mathrm{M}$. The values in two cases, a greater negative level of surface potential $(-20 \mathrm{mV}, O)$ and a smaller negative potential $(-1 \mathrm{mV}, 0)$, were plotted. The interaction energy was divided by the thermal kinetic energy $(k T)$ at $25^{\circ} \mathrm{C}$, where $k$ is the Boltzmann constant and $T$ is the absolute temperature. The same parameters and equation as in Fig. 2 were used for the calculation by the DLVO theory. The surface potentials of three $V$. alginolyticus strains were -0.5 to $-0.7 \mathrm{mV}$ by Ohshima's theory and -21 to $-23 \mathrm{mV}$ by the Smoluchowski equation at the ionic strength $0.05 \mathrm{M}$.

between bacterial cells and surfaces. Fig. 3 exemplifies the difference in the energy profile between two cases: a larger negative level of cell-surface potential obtained from the Smoluchowski equation and a smaller surface potential from Ohshima's theory.

For 'soft' particles such as bacterial cells, the energy barrier is less than that estimated from the Smoluchowski equation as shown in Fig. 3. At higher ionic strength, above a few tens of millimoles per litre for $V$. alginolyticus, the energy barrier becomes smaller than the thermal kinetic energy of the cells (see Fig. 2). In this case, the attachment rate of the cells to a substratum surface depends on the frequency of cell collision to the surface, hence on the swimming speed of the cells, as discussed below.

Suppose bacterial cells (concentration $c$ ) to be suspended and a glass plate immersed in a medium. When the cells travel distance $L$, on average, in a unit time without the aid of flagellum plus distance $V$ with flagellum rotation, the frequency of cell collision to the glass surface (area $S$ ) in a unit time is $c S(L+V) F_{1}$, where $F_{1}$ is the fraction of the cells moving toward the glass. If a part of the collisions, $F_{2}$, results in cell attachment to the surface, the attachment rate $A(V)$ is $c S(L+V) F_{1} F_{2}$. Division of the attachment rate of the cells swimming with speed $V$ by that with $V_{0}$ gives $A(V) / A\left(V_{0}\right)=(L+V) /\left(L+V_{0}\right)$. When $L \ll V_{0}$ and $V$, this equation reduces to:

$A(V) / A\left(V_{0}\right)=V / V_{0}$

Equation (3) means that the attachment rate increases proportionally to the swimming speed. We obtained Fig. 4 by plotting $A(V) / A\left(V_{0}\right)$ vs $V / V_{0}$ from the results of

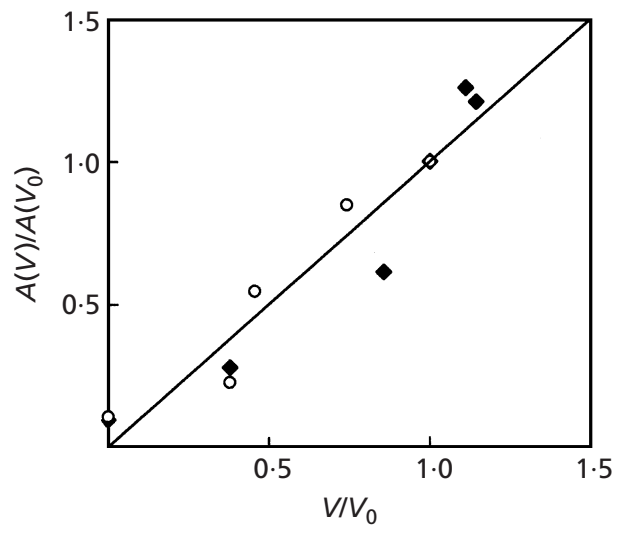

Fig. 4. Relationship between swimming speed and attachment rate of $V$. alginolyticus cells. The relative values of swimming speed and attachment rate to those at $100 \mathrm{mM} \mathrm{NaCl}$ were plotted for strain YM4. The $\mathrm{Na}^{+}$concentration was changed from 0 to $100(\bigcirc)$ or to $400 \mathrm{mM}(\diamond)$ while keeping the ionic strength at 100 or $400 \mathrm{mM}$ by adding $\mathrm{KCl}$.

Kogure et al. (1998) for $V$. alginolyticus. The relative attachment rate of strain YM4, possessing a polar flagellum rotated by $\mathrm{Na}^{+}$motive force, increased along with the relative swimming speed; we observed a similar relationship for strain NMB86, which possesses only a polar flagellum and lacks chemotactic ability (data not shown). Thus, Fig. 4 clearly indicates that the relation in equation (3) holds. This is due to the fact that the swimming cells can freely access the glass surface because of the absence of the energy barrier between them. When the rotation of the flagellum was specifically inhibited by adding amiloride, the attachment rate decreased dramatically (Kogure et al., 1998). The reduced attachment rate, although to a small extent, increased proportionally to the cell concentration of strain YM4 (data not shown). This also indicates the absence of the barrier.

When bacterial cells are non-motile, they seem to travel distance $(2 D t)^{1 / 2}$, on average, during time $t$ by Brownian motion, where $D$ is the diffusion coefficient of the cells. Thus, the number of attached cells during time $t$, $A(t)$, will be given by $A(t)=c S(2 D t)^{1 / 2} F_{1} F_{2}$, resulting in a liner relationship between the number of attached cells and the square root of the attachment time. Kogure et al. (1998) examined the time-course of cell attachment for non-motile mutants YM18 and NMB94 of V. alginolyticus, suspended in medium with different substrate concentrations. By plotting the number of attached cells against the square root of time, we obtained linear relationships for a shorter period of attachment time (data not shown). These relationships indicate the absence of the energy barrier as well as a way by which we may evaluate the value for $F_{2}$.

\section{Attachment mechanism of $\boldsymbol{V}$. alginolyticus cells}

An irreversible attachment with the premise of a high energy barrier between bacterial cells and surface has been explained as follows. A secondary energy minimum 
5-10 nm away from a substratum surface may act to halt bacterial cells long enough to result in an irreversible attachment by means of polymer fibrils, for which the electrostatic repulsion is much smaller compared with a cell, bridging the gap between the cells and surface (Rogers, 1979). However, we cannot explain the irreversible attachment of $V$. alginolyticus by this mechanism, for the following two reasons. First, the depth of the secondary minimum for the cells was not sufficient to halt the cells subject to turbulence by thermal kinetic energy. The depth was about $-1 \mathrm{kT}$ even at $400 \mathrm{mM}$, the highest electrolyte concentration examined, the calculation being based on the DLVO theory by using the same parameters as Fig. 3 and a bacterial surface potential of $-20 \mathrm{mV}$. Second, the three V. alginolyticus strains are mutants lacking lateral flagella. The polar flagellum also had no role as the bridging polymer, because the attachment rate was comparable between a strain (NMB94) having a non-rotating flagellum and a strain (YM18) without a polar flagellum (Kogure et al., 1998). The high energy barrier preventing the cells from approaching closely to a surface does seem to be present under low ionic strength conditions even for a 'soft' particle (see Fig. 2). However, under conditions of high ionic strength, the barrier becomes less than the thermal kinetic energy of the bacterial cells when the cells are rich in polymers at their surfaces, as noted by Kogure et al. (1998). Thus, we must reconsider the attachment mechanism of bacterial cells under high ionic strength conditions such as in seawater.

\section{Cell attachment of other kinds of bacteria}

We examined the relationship between the cell attachment rate to the glass surface and the swimming speed for other bacterial strains, Alcaligenes spp. 172 and 201, Alteromonas sp. WB30 and Pseudomonas aeruginosa. As observed for V. alginolyticus, the EPM of these strains did not fall to zero but approached a certain negative value at high ionic strength (data not shown), indicating the existence of a polymer layer at their cell surfaces. For all these strains, the electrostatic energy barrier becomes less than the thermal kinetic energy at ionic strengths above $70-80 \mathrm{mM}$. At an ionic strength of $100 \mathrm{mM}$ (maintained by varying the addition of $\mathrm{KCl}$ while $\mathrm{Na}^{+}$concentration was varied), as shown in Fig. 5, the attachment rate increased along with the swimming speed for Alcaligenes sp. 201 and Alteromonas sp. WB30 as well as $V$. alginolyticus YM4. It is noteworthy that the increment in the attachment rate of these two strains appears to be greater than that of V.alginolyticus YM4. The factor $F_{2}$, the fraction of cells resulting in attachment after collision to the glass surface, may be greater for these strains compared with $V$. alginolyticus YM4. In contrast, $P$. aeruginosa and Alcaligenes sp. 172 hardly attached to the glass surface and showed no such linear relation between the attachment rate and the swimming speed. The cell surfaces of these strains may have a low affinity, and thus a small $F_{2}$ value, for the glass surface. This may make it difficult to observe the relationship between the attachment rate and the

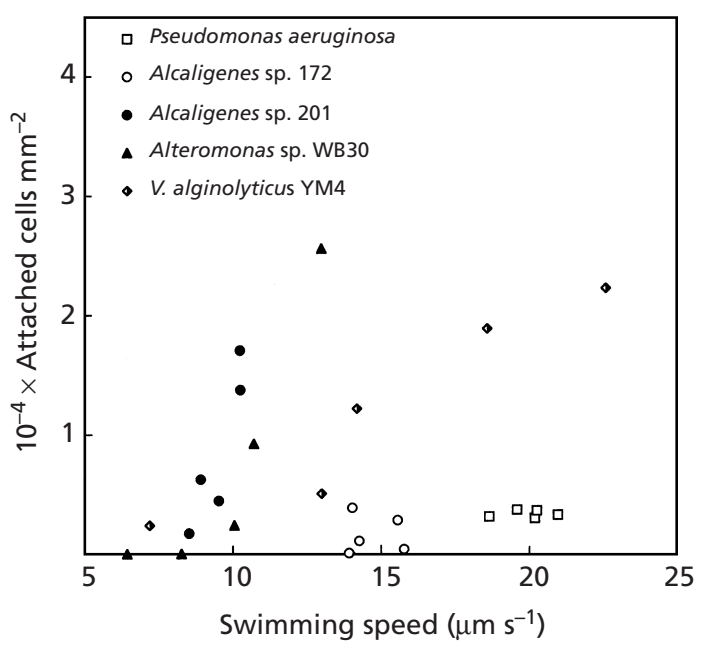

Fig. 5. Relationship between bacterial swimming speed and attachment rate to a glass surface. The $\mathrm{Na}^{+}$concentration was shifted from 0 to $100 \mathrm{mM}$ to change the bacterial swimming speed while keeping the total concentration of $\mathrm{Na}^{+}$and $\mathrm{K}^{+}$at $100 \mathrm{mM}$ by adding $\mathrm{KCl}$. The swimming speed (mean of two independent experiments measured for 20 randomly chosen cells) and attachment rate (mean of two independent experiments counting attached cell number at $25^{\circ} \mathrm{C}$ during $1 \mathrm{~h}$ ) were obtained by the same methods as previously reported (Kogure et al., 1998). Note that due to water movement during the observation and/or an unavoidable error in pointing to a specific cell during the image-analysing process, the cells seemingly swam at a speed of $6-7 \mu \mathrm{m} \mathrm{s}^{-1}$ even at $0 \mathrm{mM} \mathrm{Na}^{+}$.

swimming speed. Except for these cases, the attachment of the bacterial cells seems to be facilitated by the cell motility.

\section{Conclusions}

The present study clarified that we must take the effect of polymers at the bacterial cell surface into account to estimate the cell-surface potential correctly. This is also true for other biological cells, e.g. human erythrocytes, having polymers at their surfaces (Kawahata et al., 1990). On the other hand, solid surfaces in natural environments are usually modified by attachment of materials, mainly polymers, to form a conditioning surface before biofilm development occurs (Korber et al., 1995). The polymer layers at these surfaces will reduce the surface potential, and hence the electrostatic energy barrier, especially under conditions of high ionic strength. Thus, we must carefully reconsider the attachment mechanism of biological cells to 'soft' surfaces in conditions of high salt concentration such as seawater, blood and food-processing media.

\section{ACKNOWLEDGEMENTS}

The authors thank Drs I. Kawagishi and M. Homma for providing them with $V$. alginolyticus YM4, YM18 and NMB94. They are also grateful to anonymous reviewers for their help in improving this article. 


\section{REFERENCES}

Bos, R., van der Mei, H. C. \& Busscher, H. J. (1998). 'Soft-particle' analysis of the electrophoretic mobility of a fibrillated and nonfibrillated oral streptococcal strain: Streptococcus salivarius. Biophys Chem 74, 251-255.

Hjerten, S. (1967). Free zone electrophoresis. Chromatogr Rev 9 , 122-219.

Kawahata, S., Ohshima, H., Muramatsu, N. \& Kondo, T. (1990). Charge distribution in the surface region of human erythrocytes as estimated from electrophoretic mobility data. J Colloid Interface Sci 138, 182-186.

Kogure, K., Ikemoto, E. \& Morisaki, H. (1998). Attachment of Vibrio alginolyticus to glass surfaces is dependent on swimming speed. J Bacteriol 180, 932-937.

Korber, D. R., Lawrence, J. R., Lappin-Scott, H. M. \& Costerton, J. W. (1995). Growth of microorganisms on surfaces. In Microbial Biofilms, pp. 15-45. Edited by H. M. Lappin-Scott \& J. W. Costerton. Cambridge: Cambridge University Press.

van Loosdrecht, M. C. M., Lyklema, J., Norde, W., Schraa, G. \& Zehnder, A. J. B. (1987). Electrophoretic mobility and hydrophobicity as a measure to predict the initial steps of bacterial adhesion. Appl Environ Microbiol 53, 1898-1901.

Makino, K., Yamamoto, S., Fujimoto, K., Kawaguchi, H. \& Ohshima, H. (1994). Surface structure of latex particles covered with temperature-sensitive hydrogel layers. J Colloid Interface Sci 166, 251-258.

Marshall, K. C., Stout, R. \& Mitchell, R. (1971). Mechanism of the initial events in the sorption of marine bacteria to surfaces. $J$ Gen Microbiol 68, 337-348.
Ohshima, H. (1994). Electrophoretic mobility of soft particles. $J$ Colloid Interface Sci 163, 474-483.

Ohshima, H. (1995). Electrophoresis of soft particles. Adv Colloid Interface Sci 62, 189-235.

Ohshima, H., Makino, K., Kato, T., Fujimoto, K., Kondo, T. \& Kawaguchi, H. (1993). Electrophoretic mobility of latex particles with temperature-sensitive hydrogel layers. J Colloid Interface Sci 159, 512-514.

Rijnaarts, H. H. M., Norde, W., Bouwer, E. J., Lyklema, J. \& Zehnder, A. J. B. (1995). Reversibility and mechanism of bacterial adhesion. Colloids Surf B: Biointerfaces 4, 5-22.

Rogers, H. J. (1979). Adhesion of microorganisms to surfaces: some general considerations of the role of the envelope. In Adhesion of Microorganisms to Surfaces, pp. 29-55. Edited by D. C. Ellwood, J. Melling \& P. Rutter. London: Academic Press.

Sonohara, R., Muramatsu, N., Ohshima, H. \& Kondo, T. (1995). Differences in surface properties between Escherichia coli and Staphylococcus aureus as revealed by electrophoretic mobility measurements. Biophys Chem 55, 273-277.

Takashima, S. \& Morisaki, H. (1997). Surface characteristics of the microbial cell of Pseudomonas syringae and its relevance to cell attachment. Colloids Surf B: Biointerfaces 9, 205-212.

van der Wal, A., Minor, M., Norde, W., Zehnder, A. J. B. \& Lyklema, J. (1997). Electrokinetic potential of bacterial cells. Langmuir 13, 165-171.

Received 19 January 1999; revised 7 June 1999; accepted 29 June 1999. 